\title{
Raphael Eduard Liesegang
}

geb. 1. Sept. 1869 in Elberfeld; gest. 16. Nov. 1947 in Bad Homburg.

Blätter der Erinnerung von Ernst $\mathrm{K}$ üster.

Daten aus R. E. Liesegangs Leben:

Dr. phil. h. c. (Gießen 1919) und Dr. med. h. c. (Frankfurt a. Main). Mitglied des Kaiser-Wilhelm-Instituts für Biophysik in Frankfurt a. Main seit 1921 .

Leiter des Instituts für Kolloidforschung in Frankfurt a. Main, später Bad Homburg - seit 1937.

Mitglied der Leopold.-Carolin. Deutschen Akademie der Naturforscher in Halle (Saale).

R. E. Liesegang war Verfasser der folgenden naturwissenschaftlichen, in Buchform erschienen Werke:

Beiträge zum Problem des elektrischen Fernsehens.

Düsseldorf 1891. - 2. Aufl. 1899. - Russische Ausgabe 1895.

Photographische Chemie. Düsseldorf 1898.

Uber die Schichtungen bei Diffusionen. Leipzig 1907.

Chemische Reaktionen in Gallerten. Düsseldorf 1898. -

2. Aufl. Dresden-Leipzig 1924.

Elektrolyse in Gallerten. Düsseldorf 1899.

Geologische Diffusionen. Dresden-Leipzig 1913.

Die A chate. Dresden-Leipzig 1915.

$\mathrm{K}$ ollo id che mie 1914 b is 1922 (Wissenschaftliche Forschungsberichte

Bd. 6). Dresden-Leipzig 1922. - 2. Aufl. 1926.

Kolloideinder Technik (Wissenchaftl. Forschungsberichte Bd. 9).

Dresden-Leipzig 1923. - 2. Aufl. 1943.

Biologis che Kolloid chemie (Wissenschaftl. Forschungsberichte Bd. 20). Dresden-Leipzig 1928.

Kolloidchemische Technologie. Dresden-Leipzig 1927. 2. Aufl. 1932.

Medizinische Kolloidlehre, - hrsg. v. Lichtwitz, Liesegang

u. Spiro. Dresden-Leipzig 1935. - 2. Aufl. in Vorbereitung.

Kolloidfibel für Mediziner. Dresden-Leipzig 1936. - 2. Aufl. 1942. - 3. Aufl. 1944. 
Raphael Eduard Liesegang war Herausgeber der „W is s e n s c h a f tlichen Forschungsberich te “ (Dresden-Leipzig. Th. Steinkopff), von welchen 57 Bände erschienen sind.

Liesegang war Mitherausgeber und Mitarbeiter der Zeitschrift für wissenschaftliche Mikroskopie (Leipzig, S. Hirzel).

Im Jahre 1933 wurde zu Frankfurt a. M. die $R$ a p h a e l-E d u a r d Liesegang-Stiftung ins Leben gerufen: „Die große wissenschaftliche und technische Bedeutung der Forschungen von Dr. Dr. h. c. Raphael Eduard Liesegang und seine sonstige unermüdliche, dem selbstlosen Dienst an Wissenschaft und Technik gewidmete Lebensarbeit veranlassen eine Mehrheit seiner Freunde, Mitarbeiter und Schüler, aus Anlaß der Vollendung seines 70. Lebensjahres, ein dauerndes Andenken an sein Lebenswerk und eine Ehrung für ihn zu schaffen." Die Raphael-EduardLiesegang-Stiftung verlieh den Raphael-Eduard-Liesegang-Preis sowie Forschungsbeihilfen zur Förderung wissenschaftlicher und wissenschaftlich-technischer Forschungen auf dem Gebiete aller Naturwissenschaften und der Medizin.

Eine Selbstdarstellung seines Lebens und Bildungsganges hat $R$. E. Liesegang in der Kolloid-Zeitschrift Bd. 49 S. 226-229 (1929) gegeben.

Vor etwa vierzig Jahren erhielt ich in der Sommerfrische eine umfangreiche Drucksachensendung - ich entnahm ihr die ersten Schriften, die mir Raphael Eduard Liesegang zu überreichen die Freundlichkeit hatte; eines der Hefte trug die handschriftliche Widmung: „Dem Psychologen in Ernst Küster" — es waren Liesegangs dramatische Dichtungen.

Liesegangs Forschungen und insbesondere das nach ihm benannte Phänomen der Liesegang'schen Ringe waren mir damals schon längst bekannt; von dem Dichter Liesegang hatte ich noch nichts gehört; den Verfasser so zahlreicher Schriften zu sehen oder zu sprechen, hatte ich noch niemals Gelegenheit gehabt. Nicht ohne Befremden blätterte ich in seinen Schriften, ohne den Grund finden zu können, der ihn gerade für diese Erzeugnisse seiner Feder besonderes Interesse bei mir voraussetzen hieb.

Geraume Zeit später wurde ich mit Liesegang persönlich bekannt; ich habe in Frankfurt mit ihm die Zeiten erlebt, in welchen die Aussicht für eines seiner Dramen das Interesse eines hervorragenden Musikers gewinnen zu können und es zum Buch einer Oper werden zu sehen, ihn freudig belebte - und ebenso die spä. 
teren Jahre, in welchen Liesegang seine poetischen Versuche nicht mehr recht gelten lassen wollte. Wenn ich trotz solcher Wandlung noch oft an sie zurückgedacht habe, so geschah es vor allem deswegen, weil sie mir das Bild vervollständigen halfen, das ich von der Persönlichkeit ihres Verfassers mir zu machen trachtete.

Ein Bild von Liesegangs wissenschaftlichen Leistungen zu entwerfen, ist bereits wiederholt versucht worden. Wir alle bewundern seine unvergleichliche Vielseitigkeit und noch mehr die spielerische Leichtigkeit, mit der er beim Schreiben wie beim Plaudern Verbindungen schlug und Verknüpfungen fand, die auch entlegene Gebiete seines Wissens in überraschender Weise miteinander verbanden - nicht anders als der Meister am Webestuhl, vor dem die Schifflein herüber-hinüberschießen, die Fäden ungesehen fließen. Wir bewundern seinen Schaffensdrang, der viele Jahrzehnte lang keine Ermüdung kannte, und den Glauben an seine ,Sendung“, den er in sich trug; wenn der Umfang seiner Arbeit uns den Eindruck einer seltenen Größe gab, so bestätigte diesen vollends die rührende Bescheidenheit, mit der der wunderbare Mann von seinen Leistungen sprach, und die Anspruchslosigkeit, mit der er hinter seinen Arbeitsgenossen und Gesprächspartnern zurückzutreten für selbstverständlich geboten erachtete. In diese Bewunderung mischte sich freilich dann und wann ein schmerzliches Bedauern, wenn wir selbst nach vieljährigem Verkehr mit ihm immer wieder nur von Liesegangs Können und Wissen, seinen neuen Einfällen und neuen Plänen zu hören bekamen und dabei so wenig über Liesegang selbst erfuhren; über ihn aber mehr zu hören, hätten wir alle, die wir in seine Nähe und in seinen Kreis gelangt waren, uns sehr gewünscht, weil wir nicht nur den Gelehrten bewunderten, sondern auch den Menschen liebten; der unermüdlich Gebende schloB aber seine Hand, wenn wir über ihn selbst einmal Kunde zu empfangen fast schon hofften. Wie gern hätten wir etwas gehört von dem, was sein Herz bewegte und sein Inneres erfüllte - vielleicht hofften wir im Stillen, ihm mit Pat oder wenigstens mit Anteilnahme helfen zu können; denn daB auch ihm, dem Fruchtbaren, nicht alle Saaten volle Ernte gebracht hatten, auch ihm nicht alle Blütenträume reifen wollten, 
konnte uns ja nicht entgehen. Aber Liesegang wich solchen Versuchen am liebsten aus und verbarg sein Inneres in unsichtigem Dunkel. Wer war eigentlich Liesegang? Wir wuBten es nicht recht und wären wohl zuweilen geneigt gewesen zu glauben, daB im Laufe der Jahre das vielgesuchte Bild sich manchesmal gewandelt hätte, wenn nicht seine Handschrift, dieser unbestechliche Spiegel des inneren Menschen, durch die Jahrzehnte hindurch mit ihrer Unveränderlichkeit uns von der Festigkeit seiner Einstellung zu den Dingen der Welt überzeugt hätte.

Auf Reisen führte mich einmal ein willkommener Zufall mit einem langjährigen Freund und Arbeitsgenossen Liesegangs zusammen: Er sprach über seinen Freund, ich über den meinigen: War es derselbe Liesegang? Ich konnte nicht alles gelten lassen, was der andere sagte, und der andere schien dem nicht völlig zustimmen zu können, was ich zu sagen für richtig hielt. „Wer kennt Liesegang?" fragte er schlieBlich.

Wenn ich in den mit Liesegang geführten Gesprächen den weitgespannten Kreis seiner wissenschaftlichen Interessen bewunderte und in derselben Stunde die Fremdheit nicht verstehen konnte, mit der er Fragen des Tages und des allgemeinen Interesses gegenüberstand oder gegenüber zu stehen sich den Anschein gab, wurde mir mehr als einmal klar, wie schwer es blieb, Liesegang zu ergründen. Mit mimosenhaftem Zartgefühl sah man ihn oftmals bei Gelegenheiten zurückschrecken, die wahrlich keinen Angriff bedeuteten. Liesegang gehörte zum Geschlecht derer, die das, was sie am tiefsten und wirksamsten bewegt, am liebsten verschweigen; cum tacent, clamant.

Keine geringere Zurückhaltung als im Zwiegespräch hat Liesegang in vielen anderen Zusammenhängen bewahrt, in der Meinung, durch solche Zurückhaltung sich das von ihm stets heiß begehrte $\mathrm{MaB}$ von persönlicher Freiheit am besten sichern zu können. Jahrelang hat er von dem Doktortitel, den ihm die Gießener Philosophische Fakultät honoris causa verliehen hatte, keinen Gebrach gemacht, und es bedurfte langer Auseinandersetzungen, um ihn auch nur zur Annahme dieser hohen Auszeichnung zu bewegen. 
Ein schwerer Irrtum wäre es, aus der Zurückhaltung und sagen wir getrost: aus der Schüchternheit, die aus Liesegangs Umgangsformen und Gesprächsführung so oft sprach, auf einen Mangel an Kraft oder an Vertrauen zum eigenen Können zu schließen. Richtig ist das Gegenteil: Liesegang war nicht nur ein Mann von unerschöpflicher Arbeitskraft; er wußte um diese Kraft; sie zu betätigen war weniger sein Ehrgeiz als seine Leidenschaft; arbeiten und andern zu helfen war ihm ein schwelgerisches Glück, über dem er alle Rauhheiten des Lebens vergaB. War seine Arbeitskraft einmal durch Krankheit bedroht - er achtete seiner Leiden kaum; sein Kraftgefühl war zu groß, als daB eine vorübergehende Einbuße an ihr ihn sonderlich bedrückt hätte. Ich habe Zeiten mit Liesegang erlebt, in welchen materielle Not ihn so schwer bedrängte, da $B$ ein anderer von ihr erdrosselt worden wäre - Liesegang lieb kein Wort der Klage jemals laut werden und setzte seine Forschungsarbeit fort - mit hungerndem Magen, aber mit flammendem Herzen. Wunderbar vollends bewährte sich seine Kraft damals, als die apokalyptischen Reiter des Weltkrieges seine Arbeitsstätte und Arbeitsmittel in Asche legten; vorbildlich hat uns Liesegang, der mehr als 70 Jahre alte Forscher, damals gezeigt, da $B$ man in des Schiffsbruchs Knirschen nicht zu zagen und seine Arbeitskraft allen Gewalten zum Trutz zu erhalten die Pflicht hat.

Zahllose Briefe hat mir Liesegang im Laufe der Jahrzehnte geschrieben - die wichtigsten und inhaltsreichsten füllten eine schwere Mappe meiner Sammlungen. Nur e in Mal, glaube ich, hat aus ihnen der wirkliche, echte Liesegang unverstellt gesprochen in dem Briefe, mit dem er mich bat, in unserm Verkehr künftighin das Sie mit dem Du zu vertauschen - wie es denn in den letzten zehn Jahren seines Lebens und unseres gemeinschaftlichen Arbeitens geschehen ist. Lauter, reicher, sprudelnder sprach der wahre echte Liesegang von sich selbst aber in den Monologen, die ich in seinen Dichtungen sehen möchte. Mag ihr literarischer Wert bescheiden sein - einen höheren bekommen sie für denjenigen, der liesegang gekannt hat und hier nun endlich einmal Liesegang von heinem Gesprächspartner in Fesseln geschlagen endlich einmal ungehindert zu Worte kommen und seine Gedanken vor uns ausbrei- 
ten sieht - einen Philosophen und einen Psychologen. Was wir auf den Seiten und zwischen den Zeilen seiner Dramen lesen, rundet uns das Bild dessen, der im Laboratorium zu uns sprach, und vervollständigt die Züge, die uns lieb und teuer waren; aus den Dichtungen Liesegangs spricht er, der im Alltag unverbrüchlich schweigsam blieb.

„Mein zweites Ich überwölbt die Flur und in den Herrlichkeiten der sinkenden Sonne jauchzt mein Schweigen". Mit diesen Worten schlieBt Liesegangs Dichtung ,Grishma“, die 1903 erschien. 